eines Organs der deutschen Justiz durch die Exekutive. Dies wird nun auch vom Europäischen Gerichtshof kritisch bewertet und bleibt eine zentrale Zukunftsfrage der deutschen Staatsanwaltschaft.

Funding Open Access funding enabled and organized by Projekt DEAL.

Open Access Dieser Artikel wird unter der Creative Commons Namensnennung 4.0 International Lizenz veröffentlicht, welche die Nutzung, Vervielfältigung, Bearbeitung, Verbreitung und Wiedergabe in jeglichem Medium und Format erlaubt, sofern Sie den/die ursprünglichen Autor(en) und die Quelle ordnungsgemäß nennen, einen Link zur Creative Commons Lizenz beifügen und angeben, ob Änderungen vorgenommen wurden.

Die in diesem Artikel enthaltenen Bilder und sonstiges Drittmaterial unterliegen ebenfalls der genannten Creative Commons Lizenz, sofern sich aus der Abbildungslegende nichts anderes ergibt. Sofern das betreffende Material nicht unter der genannten Creative Commons Lizenz steht und die betreffende Handlung nicht nach gesetzlichen Vorschriften erlaubt ist, ist für die oben aufgeführten Weiterverwendungen des Materials die Einwilligung des jeweiligen Rechteinhabers einzuholen.

Weitere Details zur Lizenz entnehmen Sie bitte der Lizenzinformation auf http://creativecommons.org/ licenses/by/4.0/deed.de.

\title{
Kiene, Claudius: Karl Spiecker, die Weimarer Rechte und der Nationalsozialismus. Eine andere Geschichte der christlichen Demokratie, 308 S., Peter Lang, Berlin
} 2020.

\section{Sebastian Elsbach}

Angenommen: 3. Dezember 2021 / Online publiziert: 24. Januar 2022

(C) Der/die Autor(en) 2022

Die Person des Zentrums- und späteren CDU-Politikers Karl Spiecker (1888-1953) - auch Carl Spiecker geschrieben - dürfte höchstens Fachleuten der deutschen Geschichte des 20. Jahrhunderts bekannt sein und auch dann wird das Wissen meist nicht allzu tief reichen. Dies ist insofern irritierend, als dass Spiecker zu Lebzeiten einer der prominentesten Vertreter der deutschen Christdemokratie war. Dass er nach seinem Tod weitgehend in Vergessenheit geriet, scheint nicht nur daran gelegen

Sebastian Elsbach $(\triangle)$

Friedrich-Schiller-Universität Jena, Jena, Deutschland

E-Mail: sebastian.elsbach@uni-jena.de 
zu haben, dass er nie in die allererste Reihe der Politik aufrücken konnte, sondern wohl auch an dem Umstand - wie es sein Biograf Claudius Kiene in einem Aufsatz formuliert - dass Spiecker schlichtweg eine ,zu demokratische Persönlichkeit“ war. Seine innerparteilichen Gegner wie insbesondere Heinrich Brüning, aber auch Konrad Adenauer, wiesen im Vergleich zu Spiecker ein nicht unproblematisches Verständnis von Wert und Funktion der liberalen Demokratie auf. Als einer der führenden Köpfe des linken Zentrumsflügels wurde Spiecker in seiner Partei auch wiederholt für seine positiven Verbindungen zur Sozialdemokratie angefeindet. Um eine angemessene Einordnung von Spieckers Wirken in der Weimarer Republik, der deutsche Exilgemeinde und der frühen Bundesrepublik geht es hingegen Kiene in seinem Debüt-Buch, welches eine erweiterte Variante seiner Masterarbeit darstellt. Dass das Werk von Umfang, Quellenauswahl und -arbeit her gesehen leicht als Dissertation hätte durchgehen können, sei dabei im Vorfeld positiv angemerkt.

Kiene versucht sich dabei nicht an einer Heldengeschichte oder einer anders gefärbten narrativen Überformung von Spieckers Biografie, sondern bietet eine fundierte und sachliche Auseinandersetzung mit den Leistungen wie den Misserfolgen des Christdemokraten, der exemplarisch für die Auf- und Umbrüche der deutschen Demokratiegeschichte in der ersten Hälfte des 20. Jahrhundert steht. Spieckers erste Station seiner politischen Karriere ab 1919 steht dabei nicht - wie man meinen könnte - im Zeichen des demokratischen Aufbruches der jungen Weimarer Republik, sondern eher für eine Schattenseite in Spieckers Biografie. Im schmutzig bis blutig geführten „Abstimmungskampf“ um Oberschlesien wirkte der bis dato als Journalist tätige Spiecker auf deutscher Seite in einer zentralen Funktion mit. Als Leiter einer nachrichtendienstlichen Behörde - der ,Stelle Spiecker“ (S. 39-49) - gestaltete Spiecker im Auftrag des preußischen Innenministeriums die deutsche Gegenpropanda mit und ließ detaillierte Berichte über die Aktivitäten der polnischen Propaganda und paramilitärischen Formationen anfertigen. In dieser Funktion konnte Spiecker ein umfangreiches und sehr langlebiges Netzwerk an politischen Kontakten knüpfen, das ihm bis in die 1950er Jahre erhalten bleiben sollte (S. 222-225). Auf die Gretchen-Frage in Bezug auf Spieckers Wirken in Oberschlesien, ob er persönlich in die von deutschen Paramilitärs begangenen Morde verwickelt war, bietet Kiene zwar keine grundlegend neue Erkenntnis. Doch kann er die Annahme erhärten, dass Spiecker wohl nicht unmittelbar in die Morde involviert war, er aber nicht die erforderliche politische Kontrolle der theoretisch ihm unterstellten Verbände durchsetzen konnte, um solche blutigen Alleingänge zu verhindern (S. $45 \mathrm{f}$.).

Dies ist ein kritischer Punkt in Spieckers Biografie, da Oberschlesien ihm zeitlebens nachhängen sollte (S. 49). Dieser etwas zu kurz geratene Abschnitt in Kienes Buch wäre durchaus ausbaufähig, da der „Abstimmungskampf“ in demokratiegeschichtlicher Hinsicht wie im Hinblick auf die Entwicklung der deutsch-polnischen Beziehungen nach wie vor offene Fragen aufwirft (So unter anderem die Frage danach, welchem Konzept von nationaler beziehungsweise oberschlesischer Identität Spieckers Propaganda folgte). Zumal die Bewertung von Spieckers Wirken besser ins Verhältnis zu seinen polnischen Gegenspielern gesetzt werden könnte; sein Counterpart - Wojciech Korfanty - hatte schließlich keine großen Skrupel politische Gewalt, selbst in Form von Morden an pro-deutschen Aktivisten, einzusetzen. Die 
Balance von Kienes Buch wäre bei einem zu starken Oberschlesien-Kapitel freilich aus dem Lot geraten.

Alle weiteren Stationen von Spieckers politischem Leben werden gleichgewichtet betrachtet und es gelingt Kiene zu zeigen, wie Spiecker trotz des Gegenwindes von rechts versuchte durch journalistisches und zivilgesellschaftliches Engagement sein Idealkonzept einer starken demokratischen „Partei der Mitte“ durchzusetzen. Seine Beziehungen zum Reichsbanner Schwarz-Rot-Gold, dessen stellvertretender Vorsitzender Spiecker für einige Jahre war (was Kiene durchaus hätte erwähnen können, S. 119), sind in diesem Licht ebenso zu sehen, wie sein Engagement im Republikanischen Reichsbund oder der Vereinigung republikanischer Presse. Sehr gelungen sind auch die Kapitel über Spieckers Remigration nach 1945 und seine Bemühungen, die Zentrumspartei als Alternative zur Adenauer-CDU aufzubauen, wobei Spiecker eng mit zentralen Zeitfiguren wie Karl Arnold zusammenarbeitete. Warum sich Spieckers Konzept einer christdemokratischen „Partei der Mitte“ angesichts der zeittypischen Polarisierung nicht hat durchsetzen können und er 1949 zur CDU übertrat, kann Kiene überzeugend erläutern und auch hiermit die im Untertitel angekündigte These einer ,,andere[n] Geschichte der christlichen Demokratie“ begründen. Gerade angesichts des aktuellen Endes der Ära Merkel, in der man durchaus eine geistige Nachfolgerin Spieckers sehen könnte, kann Kienes Arbeit somit neue Impulse zur deutschen Demokratiegeschichte im Allgemeinen wie zur Geschichte der Christdemokratie im Speziellen setzen.

Funding Open Access funding enabled and organized by Projekt DEAL.

Open Access Dieser Artikel wird unter der Creative Commons Namensnennung 4.0 International Lizenz veröffentlicht, welche die Nutzung, Vervielfältigung, Bearbeitung, Verbreitung und Wiedergabe in jeglichem Medium und Format erlaubt, sofern Sie den/die ursprünglichen Autor(en) und die Quelle ordnungsgemäß nennen, einen Link zur Creative Commons Lizenz beifügen und angeben, ob Änderungen vorgenommen wurden.

Die in diesem Artikel enthaltenen Bilder und sonstiges Drittmaterial unterliegen ebenfalls der genannten Creative Commons Lizenz, sofern sich aus der Abbildungslegende nichts anderes ergibt. Sofern das betreffende Material nicht unter der genannten Creative Commons Lizenz steht und die betreffende Handlung nicht nach gesetzlichen Vorschriften erlaubt ist, ist für die oben aufgeführten Weiterverwendungen des Materials die Einwilligung des jeweiligen Rechteinhabers einzuholen.

Weitere Details zur Lizenz entnehmen Sie bitte der Lizenzinformation auf http://creativecommons.org/ licenses/by/4.0/deed.de. 